\title{
Knowledge Representation in Noninvasive and Invasive Comprehensive Intelligent Cardiovascular Diseases Diagnosis in e-Home Healthcare
}

\author{
Tai Chun Tang, Mu Bo Chen, Bin Bin Fu, Ming Chui Dong, Zhao Xiong Fang, and Zhen Hua Xiao
}

\begin{abstract}
A cardiovascular diseases (CVDs) knowledge hierarchical representation (KHR) is pioneered to perform noninvasive and invasive comprehensive diagnosis in e-home healthcare, thus to achieve high-precise diagnostic accuracy. The clinical diagnosis actually maps the sampled cardiovascular parameters (CPs) into the relevant CVDs through using medical knowledge and depending on pathological characters of the disease. The CPs include noninvasive physical information such as electrocardiography (ECG), sphygmography (SPG), and heart sound (HS) data as well as invasive chemical information such as blood test parameters (BTPs) data. CVDs are divided into different categories with the allowance of producing new variants. Tackle such complex and diversified data, a frame-based KHR is proposed which represents the CPs, disease categories and their pathological relationships hierarchically. It is noteworthy that such a KHR is suitable to knowledge changes with the re-usable shell design structure. In addition, a versatile KHR inference mechanism (KHR-IM) is introduced to realize this KHR. With such an organic combination of noninvasive and invasive diagnosis, it establishes not only a solid mainstay for realizing more accurately the functionality of entire system, but also provides people with a reliable health monitor and management.
\end{abstract}

Index Terms-Cardiovascular diseases, cardiovascular parameters, frame-based knowledge hierarchical representation, inference mechanism, noninvasive and invasive comprehensive diagnosis, symptom-disease mapping relationship.

\section{INTRODUCTION}

Cardiovascular diseases (CVDs) are globally known as the number one killer of health [1]. For offering early prevention, and proactive detection of CVDs, various e-health applications have been flourishing, such as telemedicine, remote monitoring, and mobile health (m-health) [2]-[4].

As [5] proposed, adapting the noninvasive and invasive comprehensive intelligent CVDs diagnoses in e-home

Manuscript received April 3, 2015; revised October 23, 2015. This research work was supported in part by the Research Committee of University of Macau under Grant No. MYRG2014-00060-FST, and in part by the Science and Technology Development Fund (FDCT) of Macau S.A.R under Grant No. 016/2012/A1, respectively.

Tai Chun Tang is with the Department of Software Engineering, Faculty of Science and Technology, University of Macau, Macau S.A.R., China (e-mail: tangtai009@163.com).

$\mathrm{Mu}$ Bo Chen, Bin Bin Fu, and Ming Chui Dong are with the Department of Electrical and Computer Engineering, Faculty of Science and Technology, University of Macau, Macau S.A.R., China (e-mail: chenmubocs@gmail.com, ariespleo51@gmail.com, mcdong@umac.mo).

Zhao Xiong Fang and Li Chun Luo are with Shaoshan People's Hospital, Xiangtan, Hunan, China (e-mail: 522706981@qq.com). healthcare can guarantee high-precise diagnostic accuracy. The proposed intelligent diagnosis system includes three entities, namely upstream (hospital server), midstream (local computers), and downstream (embedded-link devices, such as smartphone, iPad, etc.), which can satisfy users with diversified backgrounds and requirements [6].

To achieve high-precise diagnostic accuracy, the integration of noninvasive e-home healthcare with invasive clinical diagnosis must be established. The clinical diagnosis actually maps the sampled cardiovascular parameters (CPs) into the relevant CVDs through using medical knowledge and depending on pathological characters of the disease. The CPs include noninvasive physical and invasive chemical information. At the aid of pervasive body sensor network, multi noninvasive signals such as electrocardiography (ECG), sphygmography (SPG), and heart sound (HS) waveforms can be obtained. Through signal pre- and post-processing, the sampled waveforms are converted into noninvasive parameters (NiP). With the aid of hospital clinical biochemical detection, multi invasive blood test parameters (BTPs) such as total cholesterol (TCH) and aspartate (AST) etc., which are a-part-of invasive parameters (InP), can be obtained. Noninvasive or invasive diagnoses are actually to infer the CVDs based on patients' NiP or InP individually. The unit, scale, value-range, describing method, and storage method of these two types of parameters are quite different; consequently the knowledge representations and relevant inference machines are quite different.

Tackle the bottleneck problem of designing a knowledge hierarchical representation (KHR) scheme, and expressing such complex and diversified data, many aspects must be considered systematically and scientifically. For instance, the confirmation of some diseases may refer to other new CPs such as creatine kinase (CK), or other relevant parameters such as brain wave parameters (BWPs), ultrasound parameters, lung-heart parameters, etc. Moreover, CVDs are divided into different categories with the allowance of producing new variants. For example, in the coronary heart disease (CHD) category it may produce a new disease called as latent coronary heart disease $(\mathrm{LCH})$.

In this paper, a frame-based KHR with re-usable shell structure [7] is proposed which is expandable and adaptable for representing such complex and diversified data. In addition, a versatile KHR inference mechanism (KHR-IM) is introduced to realize this KHR.

\section{MethodolOGY}

\section{A. Knowledge Hierarchical Representation}




\section{1) Architecture of KHR for cardiovascular parameters}

The designed architecture of KHR for CPs is illustrated in Fig. 1, in which four-layer parameter categories are concerned, namely, grand-parent, parent, child and grand-child.

In detailed, the hemodynamic parameters (HDPs) which are derived from noninvasive signals SPG waveforms, can be divided into three child parameter categories: sensitive (SSE), auxiliary (AXY), and replenish (RPH) according to their diagnostic importance [8].

Similarly, BTPs can be categorized into several child parameter categories such as blood lipid parameters (BLPs), myocardial enzyme parameters (MEPs), liver function parameters (LFPs) and renal function parameters (RFPs), which are the indicators to the metabolism of cardiovascular, liver and renal function respectively. Clinically, those parameters are quite sensitive for CVDs diagnosis.

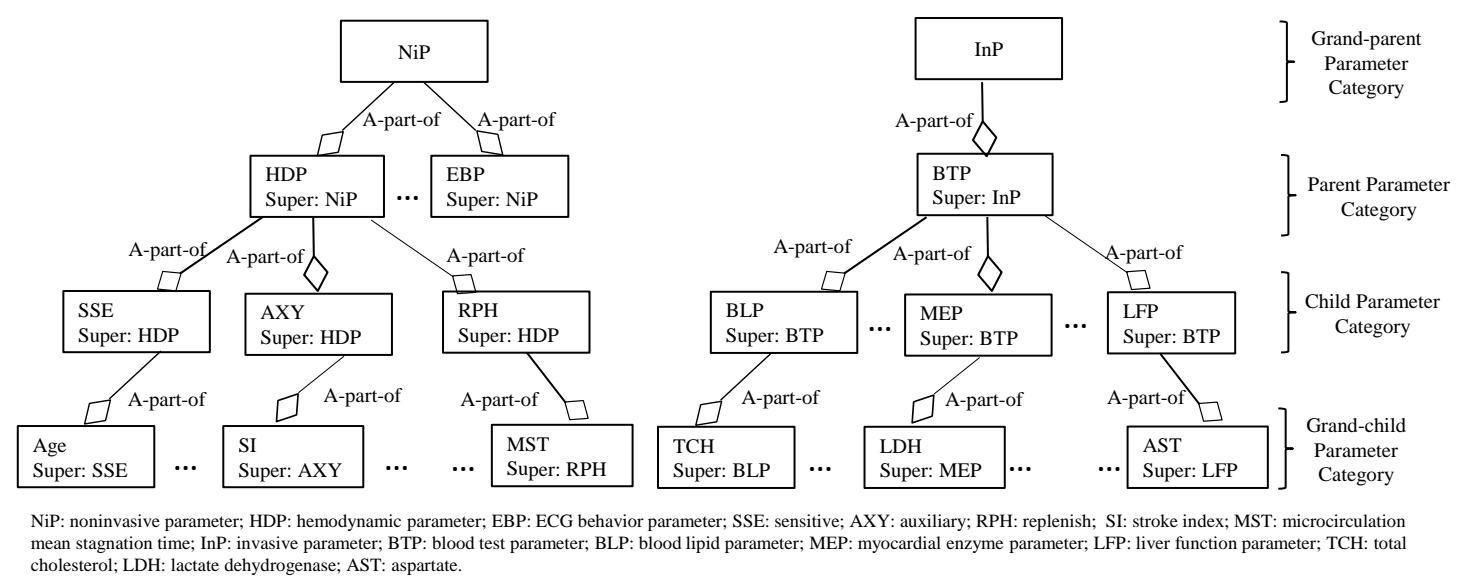

Fig. 1. Architechture of KHR for CP.

\begin{tabular}{|l|}
\hline Cardiovascular Parameter (CP) \\
\hline Frame: BTP in KHR \\
Superclasses: CP.InP \\
Subclasses: BLP, MEP, LFP, RFP, ... \\
SLOT: \\
Name: Blood test parameter \\
Unit: \#string \\
Scale: \#string \\
//Normal-value-range (NVR) \\
NVR-Max-Children: \#string \\
NVR-Min-Children: \#string \\
NVR-Max-Adult: \#string \\
NVR-Min-Adult: \#string \\
Store-method: \#string \\
.. \\
\\
Frame: TCH in KHR \\
Superclasses: CP.InP.BTP.BLP \\
Subclasses: None \\
SLOT: \\
Name: Total cholesterol \\
Unit: mmol/L \\
Scale: mmol/L×38.67 $\rightarrow$ mg/dL \\
NVR-Max-Children: 5.20 \\
NVR-Min-Children: 3.12 \\
NVR-Max-Adult: 6.00 \\
NVR-Min- Adult: 2.9 \\
Store-method: double \\
.. \\
\end{tabular}

\begin{tabular}{|l|}
\hline Disease Set (DS) \\
\hline Frame: CHD in KHR \\
Superclasses: DS.CVD \\
Subclasses: UAA, NST, STE, ... \\
SLOT: \\
Name: Coronary heart disease \\
Clinical manifestations: \#string \\
Suggestion: \#string \\
$\ldots$ \\
\\
Frame: LCH in KHR (disease variants) \\
Superclasses: DS.CVD.CHD \\
Subclasses: None \\
SLOT: \\
Name: latent coronary heart \\
disease \\
Clinical manifestations: breathing \\
difficulty, chest tightness, ... \\
Suggestion: go to the hospital for \\
further testing, ... \\
$\ldots$
\end{tabular}

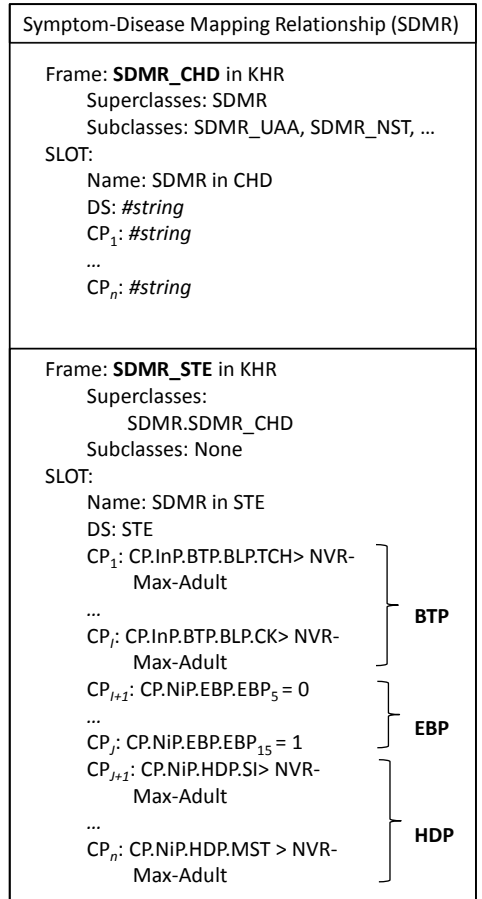

Fig. 2. (a) Frame instance of CP; (b) Frame instance of DS; (c) Frame instance of SDMR.

Accordingly, as an example of frame construction, Fig. 2 (a) shows the BTP frame and its subclass TCH frame. As aforementioned, chemical InP and physical NiP have diverse attributes like unit, scale, value-range, describing method, and storage method, frames can incorporate sets of attribute descriptions using slots. As illustrated in bottom of Fig. 2 (a), a frame representing the TCH class might have slots for those different attributes, which proves that the frame-based KHR is adaptable flexibly for representing such complex and diversified data.

Another advantage by applying frame-based KHR lies in its expandability. For instance, the confirmation of some diseases may refer to other new $\mathrm{CP}$ such as $\mathrm{CK}$, or other relevant parameters such as BWP, ultrasound parameters, and lung-heart parameters etc. Once the symptom-disease mapping relationship (SDMR) between the physical BWP with CVDs established, the new BWP frame can be created easily by only automatically inheriting all attributes of the above parent NiP frame.

\section{2) Architecture of KHR for Disease Set (DS)}

The designed architecture of KHR for DS is illustrated in Fig. 3, in which three-layer categories are concerned, namely, grand-parent, parent, and child. The child diseases unstable angina (UAA) and ST-segment elevation myocardial 
infarction (STE) are categorized under the parent disease CHD. When a new disease called as LCH meets the CHD diagnostic criteria, it will also be categorized under CHD shown as the dash arrow and blocks.

In practical frame realization, a frame inherits the attributes of all parent class frames to which it links. For example, as illustrated in bottom of Fig. 2 (b), the frame LCH would acquire coincidently three slots Name, Clinical manifestations, and Suggestion from the above parent CHD frame apart from creating its own slots. Hereby, this frame-based KHR shows good reusability and adaptable to new disease variants.

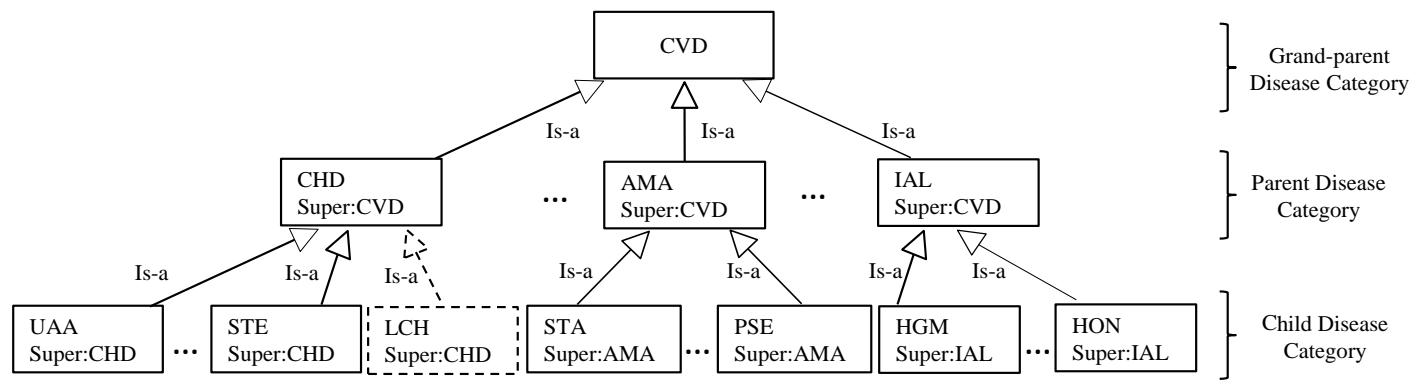

CVD: cardiovascular disease; CHD: coronary heart disease; AMA: cardiac arrhythmia; IAL: ion abnormal; UAA: unstable angina; STE: ST-segment elevation myocardial infarction; LCH: latent coronary heart disease; STA: sinus tachycardia; PSE: preexcitation syndrome; HGM: hyperglycemia; HON: hyponatremia.

Fig. 3. Architechture of KHR for DS.

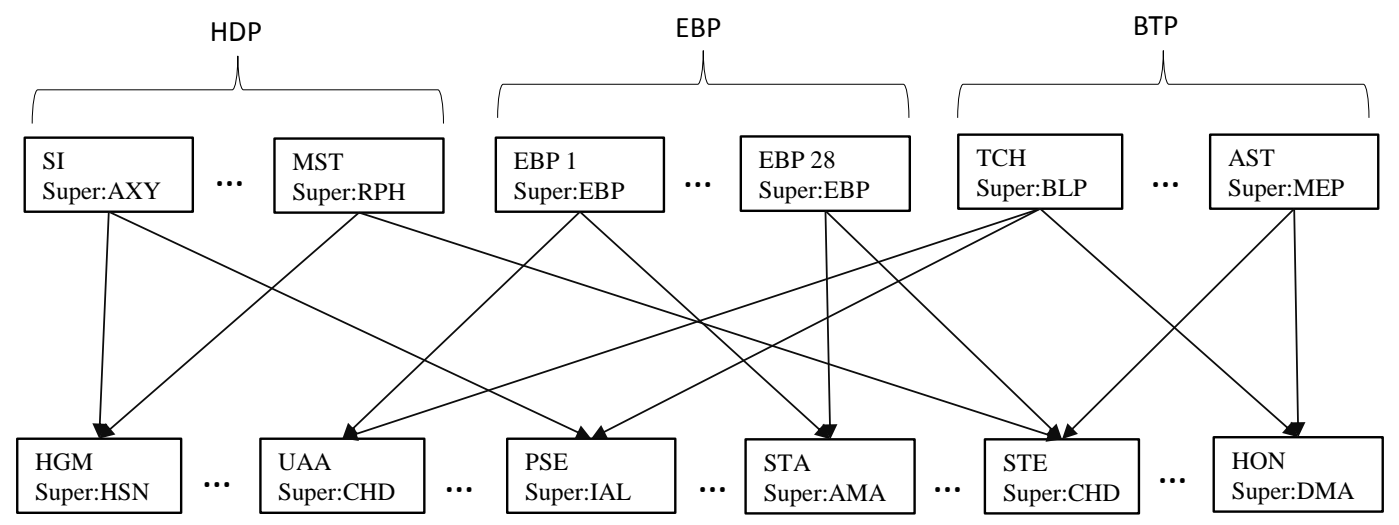

Fig. 4. Architechture of KHR for realizing SDMR.

\section{3) Architecture of KHR for realizing the} symptom-disease mapping relationship

The designed architecture of KHR for realizing SDMR is illustrated in Fig. 4. Actually, some diseases may relate only with HDP, ECG behavior parameter (EBP), or BTP. For instance, deduce the category hyperglycemia (HGM) needs only utilizing physical HDP. However, diagnosing some diseases like STE may refer to all the physical HDP, EBP and the chemical BTP.

The corresponding design of the SDMR_CHD frame and its subclass SDMR_STE are shown in Fig. 2 (c).

\section{B. Inference Mechanism}

A versatile KHR-IM is originated to verify this KHR. Patients' CPs are operated through a clinical inference function $\boldsymbol{F}(\mathrm{x})$ to determine whether the reflected cardiovascular status is normal or abnormal.

In detailed, the operation of $\boldsymbol{F}(\mathrm{x})$ can be summarized in the following three steps:

Firstly, $h$ CPs are acquired which include $a$ physiological parameters (PLPs), $b$ BTPs, $c$ EBPs along with $d$ HDPs, where $h, a, b, c, d$ denote numbers. The CPs are filled into the CPs record matrix $\mathbf{P}_{k \times h}$ as:

$$
\mathbf{P}=\left[\begin{array}{cccc}
p_{1,1} & p_{1,2} & \cdots & p_{1, h} \\
p_{2,1} & p_{2,2} & \cdots & p_{2, h} \\
\vdots & \vdots & \ddots & \vdots \\
p_{k, 1} & p_{k, 2} & \cdots & p_{k, h}
\end{array}\right]_{k \times h}
$$

where $k$ is the total number of patients to be diagnosed. Thus each row in $\mathbf{P}_{k \times h}$ is one CPs record for a patient and specifically $p_{x, q}$ is the $x^{\text {th }}$ patient's $q^{\text {th }} \mathrm{CP}$.

Secondly, $\mathbf{P}_{k \times h}$ is mapped to parent disease canonical matrix $\mathbf{C}_{h \times w}$ such that the reflected parent disease could be recognized. The $\mathbf{C}_{h \times w}$ is expressed as:

$$
\mathbf{C}=\left[\begin{array}{cccc}
c_{1,1} & c_{1,2} & \cdots & c_{1, w} \\
c_{2,1} & c_{2,2} & \cdots & c_{2, w} \\
\vdots & \vdots & \ddots & \vdots \\
c_{h, 1} & c_{h, 2} & \cdots & c_{h, w}
\end{array}\right]_{h \times w}
$$

where $w$ is the total number of parent disease categories. The parent disease diagnostic results matrix $\mathbf{I D}_{k} \times{ }_{w}$ is illustrated in:

$$
\mathbf{I D}=\mathbf{P} \times \mathbf{C}=\left[\begin{array}{cccc}
i d_{1,1} & i d_{1,2} & \cdots & i d_{1, w} \\
i d_{2,1} & i d_{2,2} & \cdots & i d_{2, w} \\
\vdots & \vdots & \ddots & \vdots \\
i d_{k, 1} & i d_{k, 2} & \cdots & i d_{k, w}
\end{array}\right]_{k \times w}
$$

where $i d_{y, s}\left(i d_{y, s}=0\right.$ or $\left.i d_{y, s}=1\right)$ is diagnostic result of the $s^{\text {th }}$ parent disease for the $y^{\text {th }}$ patient. $i d_{y, s}=0$ represents the $y^{\text {th }}$ patient does not have the $s^{\text {th }}$ parent disease, $i d_{y, s}=1$ means the $y^{\text {th }}$ patient has $s^{\text {th }}$ parent disease.

Finally, $\mathbf{I D}_{k} \times{ }_{w}$ is mapped to child disease canonical matrix $\mathbf{D C}_{w} \times{ }_{n}$ such that the final disease is indicated. The matrix 
$\mathbf{D C}_{w} \times{ }_{n}$ is expressed as:

$$
\mathbf{D C}=\left[\begin{array}{cccc}
d c_{1,1} & d c_{1,2} & \cdots & d c_{1, n} \\
d c_{2,1} & d c_{2,2} & \cdots & d c_{2, n} \\
\vdots & \vdots & \ddots & \vdots \\
d c_{w, 1} & d c_{w, 2} & \cdots & d c_{w, n}
\end{array}\right]_{w \times n}
$$

where $n$ is the total number of child disease categories. The child disease diagnostic result matrix $\mathbf{D}_{k} \times{ }_{n}$ is shown in:

$$
\mathbf{D}=\mathbf{I D} \times \mathbf{D C}=\left[\begin{array}{cccc}
d_{1,1} & d_{1,2} & \cdots & d_{1, n} \\
d_{2,1} & d_{2,2} & \cdots & d_{2, n} \\
\vdots & \vdots & \ddots & \vdots \\
d_{k, 1} & d_{k, 2} & \cdots & d_{k, n}
\end{array}\right]_{k \times n}
$$

where $d_{z, t}\left(d_{z, t}=0\right.$ or $\left.d_{z, t}=1\right)$ is the final diagnostic result of the $t^{\text {th }}$ child disease for the $z^{\text {th }}$ patient.

In summary, by applying such versatile KHR-IM, the KHR which represents the CPs, relevant CVDs and their pathological relationships can be verified.

\section{EXPERIMENTAL RESULTS}

For the traditional inference mechanism (T-IM), the entire $n$ rules for a specific patient are checked one by one. Thus the computation complexity is $\mathrm{O}(n)$.

By contrast, the KHR-IM employs hierarchical diagnosis strategy by checking parent diseases firstly and then going to the corresponding child diseases if parent disease is positive. Therefore, the computation complexity is $\mathrm{O}(w+n / w)$.

To evaluate diagnostic performance of KHR-IM, here the improved computation complexity $(I C C)$ is defined as:

$$
I C C=\left(1-\frac{O\left(w+\frac{n}{w}\right)}{O(n)}\right) \times 100 \%
$$

where $w$ is the total number of parent diseases, and $n$ is the total number of child diseases, as depicted in (2) and (4) respectively.

In this work, totally 103 CPs including 7 PLPs, 36 BTPs, 28 EBPs along with 32 HDPs are employed to diagnose 5 most common types of parent diseases and 30 types of child diseases; thus $h=103, a=7, b=36, c=28, d=32, w=5$ and $n=30$. The testing results show that ICC of the proposed KHR-IM is $63.33 \%$ compared with T-IM by (6).

Higher ICC indicates better performance in terms of the resource and memory reduction.

\section{CONCLUSION}

A frame-based KHR is pioneered to perform noninvasive and invasive comprehensive CVDs diagnosis in e-home healthcare efficiently. KHR represents the complex and diversified data include CPs, disease categories and their pathological relationships hierarchically. It is noteworthy that such a KHR is suitable to knowledge changes with the reusable shell design structure. In addition, a versatile KHR-IM is introduced and verified that KHR has good performance in decreasing the amount of resource and memory.

\section{ACKNOWLEDGEMENT}

The author would like to thank to Research Committee of University of Macau under Grant No. MYRG2014-00060-FST, the Science and Technology Development Fund (FDCT) of Macau S.A.R under Grant No. 016/2012/A1 and Mr Min Xiao give the support to this paper.

\section{REFERENCES}

[1] S. Mendis, P. Puska, and B. Norrving, "Global atlas on cardiovascular disease prevention and control," World Health Organization, 2011.

[2] M. A. Fengou, G. Mantas, D. Lymberopoulos, N. Komninos, S. Fengos, and N. Lazarou, "A new framework architecture for next generation e-health services," IEEE Journal of Medical and Health Informatics, vol. 17, no. 1, pp. 9-18, 2013.

[3] E. Kyriacou, S. Pavlopoulos, A. Berler, M. Neophytou, A. Bourka, A. Georgoulas, D. Koutsouris et. al., "Multi-purpose healthcare telemedicine systems with mobile communication link support," Biomedical Engineering Online, vol. 2, no. 7, 2003.

[4] A. Sasan, "Link technologies and blackberry mobile health (mhealth) solutions: a review," IEEE Trans. on Info. Tech. in Biomedicine, vol. 16, no. 4, pp. 586-597, 2012.

[5] T. C. Tang, M. B. Chen, J. L. Ma, M. C. Dong, Z. X. Fang, and L. C. Luo, "Noninvasive and invasive comprehensive intelligent cardiovascular diseases diagnosis in e-home healthcare," International Journal of Bioscience, Biochemistry and Bioinformatics (IJBBB), vol. 4, no. 5, pp. 326-330, 2014.

[6] J. L. Ma and M. C. Dong, "R\&D of versatile distributed e-home healthcare system for cardiovascular disease monitoring and diagnosis," presented at IEEE-EMBS International Conference on Biomedical and Health Informatics, Valencia, Spain, 2014.

[7] R. J. Brachman, R. E. Fikes, and H. J. Levesque, "KHRypton: A functional approach to knowledge representation," Computer, vol. 16, no. 10 , pp. 67-73, 1983.

[8] J. Shi, B. D. Sekar, M. C. Dong, and X. Y. Hu, "Extract Knowledge from Site-sampled Data Sets and Fused Hierarchical Neural Networks for Detecting Cardiovascular Diseases," Biomedical Engineering and Biotechnology (iCBEB), pp. 275-279, May 2012.

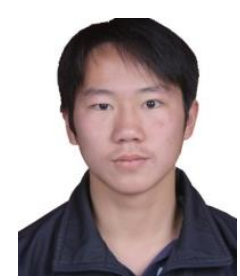

Tai Chun Tang received the master degree in in software engineering in 2015 from University of Macau, Macau, China. He received the bachelor degree in computer science and technology in 2012 from Shihezi University, Sinkiang, China. His current research is oriented to R\&D 3-Rank CVD intelloigent diagnosis.

His main research interests are machine learning, application of AI in biomedical engineering and similarity and complexity analysis.

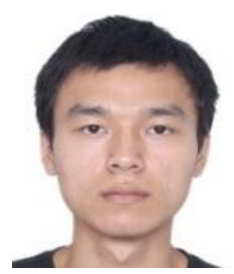

Mu Bo Chen is currently pursuing the MSc degree in ECE Department of University of Macau, Macau. His current research is oriented to $R \& D$ a hybrid intelligent system to diagnose cardiovascular disease. $\mathrm{He}$ received Bachelor degree in CS in 2012 from Guangdong University of Foreign Studies.

His research interests include machine learning, applying AI technology in various engineering and biomedical applications etc.

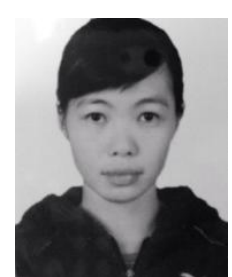

Bin Bin Fu is currently pursuing the $\mathrm{PhD}$ degree in ECE Department of University of Macau, Macau.

Her research interests focus on embedded system, biomedical signal processing and artificial intelligence, towards the goal of synthesizing them for wearable/portable monitoring with computerized risk stratification.

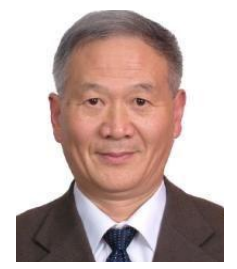

Ming Chui Dong is full professor and $\mathrm{PhD}$ supervisor of ECE Department of University of Macau, Automation Department of Tsinghua University, China and a professor of YanTai University, China. He received the MSc degree in EEE in 1975 at Tsinghua University, China, a visiting scholar in EEE in 1981 at Rome University, Italy.

His main research interests are AI and its application 
in biomedical engineering, CIMS, fault diagnosis, AI in vision-to-text, voice-to-text machine translation etc.

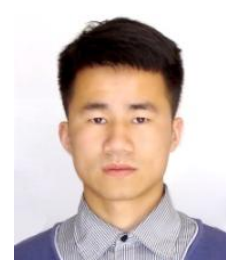

Zhao Xiong Fang is currently a doctor of the Emergency Department, Shaoshan People's Hospital, Xiangtan, Hunan, China. He received the bachelor degree in Department of Clinical Medicine in 2012 from University of South China. His research interests include cardiovascular disease, cerebral vessels disease diagnosis and disease intelligent diagnosis.
Zhen Hua Xiao is currently director of the Emergency Department, Shaoshan People's Hospital, Xiangtan, Hunan, China. He is with the Department of Clinical Medicine from Xiangya School of Medicine, Central South University. His research interests include cardiovascular disease, cerebral vessels disease diagnosis and disease intelligent diagnosis. 